\section{Dealing with data: a milestone in statistical genetics}

\author{
Handbook of Statistical Genetics \\ DJ Balding, M Bishop and C Cannings \\ John Wiley E Sons, Inc., New York. 2001; 863 pp. \\ $£ 175.00$, hardback. ISBN 0-471-86094-8 \\ Heredity (2003) 91, 1. doi:10.1038/sj.hdy.6800274

\section{Reviewed by KE Holsinger}

The world of genetics has seen an explosion of data with gene sequences and whole genomes accumulating in databases at an astounding rate. Fortunately, it has also seen an explosion of interest in developing the statistical tools necessary to analyze and understand these data. Unfortunately, the tools have been widely scattered, difficult to find, and almost impossible to compare with one another. The editors of this book have done us a great service by inviting analytical reviews of nearly all relevant topics from leaders in the field and making them available in a single, admittedly very large, volume. The tools available can now be easily identified, their possibilities and limitations assessed.

The Handbook of Statistical Genetics provides a comprehensive reference to methods of statistical genetics ranging from chromosome mapping, sequence comparison, and gene prediction through coalescent theory and population structure analysis to phylogenetics, genetic epidemiology, plant and animal breeding, forensics, and conservation genetics. No other single volume covers the field so comprehensively, and no other single volume gathers such a distinguished list of contributors between its covers. At 863 pages and $£ 175.00$, I cannot suggest that it belongs on the shelf of every geneticist, but it certainly belongs on the shelf of every library in colleges, universities, and institutes that have geneticists on their staff.

Each of the 30 chapters is written by a distinguished geneticist, and the editors and publishers allowed each of them the space they needed for comprehensive, analytical reviews. To focus on examples from areas I know best, Neuhauser provides a wonderfully lucid account of basic models in population genetics, and Nordborg provides a masterful overview of coalescent theory that is complemented by Stephens' review of likelihood and Bayesian approaches for statistical inference using coalescent theory. Rousset contributes a rigorous overview of inference approaches from spatially structured populations, Excoffier gives a thorough account of the use and interpretation of statistics related to partitioning genetic variation within and among populations, and Hudson concludes the section of the book dealing with population genetics with a thorough review of gametic disequilibrium and its properties under different population genetic models. Each of these chapters both summarizes the current state of the art and provides an excellent collection of important references for those readers who want to delve further into the topics that are treated.

Unlike many edited volumes, especially edited volumes with 30 chapters, the quality of every contribution is very high. But be forewarned. This is a book on statistical genetics. While some chapters discuss their topics with references to only a few equations that fit on single lines (eg, Brookfield's fascinating chapter on genome evolution), most have a large dose of conditional probability, expectation, MCMC computational methods, diffusion approximations, or other mathematically sophisticated techniques. Each of the authors has done an excellent job in reducing the complexity of the field to the extent that they can, but most of the topics dealt with in the book are inherently statistical and often inherently complex. None of the chapters are easy to read, but all will repay close reading.

The Handbook of Statistical Genetics is sure to become the standard reference for students and researchers in all fields of statistical genetics. The individual chapters provide some of the best analytical reviews of the various subfields of statistical genetics to be found anywhere, and the book as a whole provides the most comprehensive overview of the field currently available. The Handbook of Statistical Genetics is one of those rare commodities, an edited volume whose intellectual value may actually increase with time.

KE Holsinger

Department of Ecology E Evolutionary Biology U-3043 University of Connecticut Storrs CT 06269-3043 USA

E-mail: kent@darwin.eeb.uconn.edu 